Interactive comment on "A N-dimensional Fortran Interpolation Program (NterGeo.v2020a) for Geophysics Sciences - Application to a back-trajectory program (BACKPLUMES.v2020r1) using CHIMERE or WRF outputs" by Bertrand Bessagnet et al.

\title{
Bertrand BESSAGNET
}

bertrand.bessagnet@Imd.polytechnique.fr

Received and published: 15 July 2020

See Supplement document 
Interactive comment on Geosci. Model Dev. Discuss., https://doi.org/10.5194/gmd-2020-88, 2020.

Interactive

comment 